\title{
Aspekte sehen und religiöser Glaube
}

Hartmut von Sass

Nicht alles, was wir wahrnehmen, nehmen wir perspektivisch wahr. Sicher beobachten wir immer schon von einem gewissen Standpunkt, beziehen uns dabei auf einen bereits eingegrenzten Bereich und tun dies unter einer bestimmten Hinsicht, also notwendig selektiv. ${ }^{1}$ Schauen wir etwa im Fernsehen Fussball, legt die Kamera den terminus a quo fest, zeigt uns einen Ausschnitt des Spielfeldes und konzentriert sich dabei zumeist auf die ballführende Spielerin. Sässen wir selbst im Stadion, änderte sich daran zwar Einiges, nicht aber Entscheidendes.Von Perspektiven zu sprechen, hat jedoch nur dann Sinn, wenn es möglich ist, etwas auch auf andere Weise sehen zu können. Insofern existieren Perspektiven nur in der Mehrzahl oder umgekehrt: Gäbe es nur eine Perspektive, wäre sie keine. Im Fussball ist der Plural der Perspektiven schon sprichwörtlich. Anders liegen die Dinge auf davon zu unterscheidenden (Spiel)Feldern.

Dies lässt sich verdeutlichen anhand einer doppelten Differenzierung, die in das Anders-Sehen-Können einzutragen ist: erstens mit Blick auf die Art des hier relevanten (Nicht)Könnens, zweitens hinsichtlich des Gegenstandsbereiches, der gesehen wird. Es geht also um die modi des Sehens einerseits und des Gesehenen andererseits. Beginnen wir mit zweitem. Offensichtlich können wir einen Sachverhalt unter moralischen, religiösen oder gar sportlichen Gesichtspunkten ansehen, auch als Witz, ernsthafte Replik oder versteckte Frage (auf etwas als etwas Bezug nehmen). Genauso können wir uns unter bestimmten Bedingungen unmittelbar auf moralische, religiöse, sportliche, witzige, ernsthafte oder fragende Sachverhalte beziehen (auf etwas Bezug nehmen). Die Als-Struktur kann nun in den direkten Bezug integriert werden, sodass wir beispielsweise einen religiösen Sachverhalt als moralisch entscheidenden oder ernsthaft gemeinten empfinden. Doch diese Integration hat offenbar ihre Grenzen, womit wir zum zweiten Moment kommen; denn häufig ist es Menschen unmöglich, etwas als etwas Bestimmtes anzusehen: ein religiöses Symbol als Karikatur, die Welt als Gottes Werk oder die gewaltsame Unterdrückung von Lebewesen als verbürgtes Recht. Wiederum andere kommen nicht umhin, das erste als Witz abzutun, im zweiten Fall von Gottes Schöpfung zu sprechen und im letzten ein

\footnotetext{
1 I.U. Dalferth, Kombinatorische Theologie, Freiburg i.Br. 1991, 87f - jedoch würde er wohl dem ersten Satz nicht zustimmen.
} 
verabscheuungswürdiges Verbrechen zu erkennen. An den Rändern der perspektivischen Wahrnehmung stehen folglich existentielle und/oder kognitive Unmöglichkeiten bzw. Notwendigkeiten, die in eine Lebenspraxis eingebettet sind. Ruft jemand nach Hilfe, sehen wir diesen nicht als jemanden an, der Hilfe benötigt, unter anderen Gesichtspunkten jedoch etwas anderes, sondern dieser Mensch ist jemand, der einfach um unsere Hilfe fleht. In diesem Fall müssen wir das 〈als〉 kürzen oder mit anderen Worten: Es ist die für uns in diesem Moment notwendige Perspektive - und damit gar keine.

Um zu unterstreichen, dass die Möglichkeit unterschiedlicher Perspektiven und die Notwendigkeit ihrer Grenzen ein interessantes Feld abstecken, muss man nicht behaupten, damit würde «das Humane> schlechthin bezeichnet. ${ }^{2}$ Vielmehr werden wir sehen, dass uns eine bestimmte Ausprägung, den Perspektivismus zu formulieren, auf anderen Gebieten - im vorliegenden Fall: dem theologischen - etwas Weiterführendes zu lehren vermag. Ich gehe daher auf Wittgensteins Idee des Aspekte-Sehens ein (Abschnitt I), wende diese Idee anschliessend auf den religiösen Glauben an (Abschnitt II) und widme mich zum Schluss knapp den Folgen und Grenzen dieses Manövers (Abschnitt III). Geleitet ist dieser Dreischritt von der Frage, wie religiöser Glaube im Rahmen des Aspekte-Sehens - wenn auch ganz skizzenhaft - zu charakterisieren ist, und von dem ebenso lakonischen Bekenntnis, dass das mögliche Resultat zu begrüssen ist.

\section{Aspekte-Sehen}

Zunächst das schon fast unvermeidliche Bild:

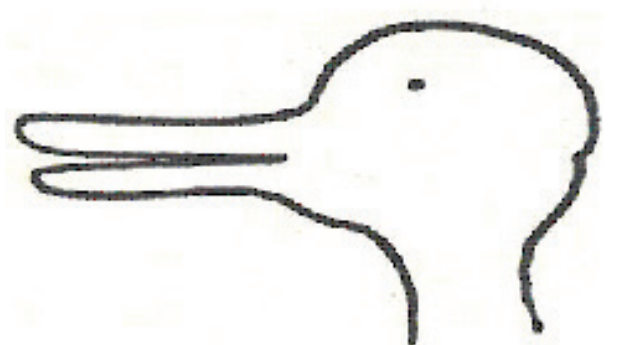

2 So S. Mulhall mit dieser exegetischen These zum reifen Wittgenstein: On Being in the World, London 1990, 4; vgl. dagegen die wohl begründete assimilatorisches These bei M. Wild, Tierphilosophie, Hamburg 2008, bes. Kap. 2.3 \& 4.2. 
Es wird Dir, lieber Leser, sicher keine Mühe bereitet haben, den Hasen und die Ente in dem ohnehin recht bekannten und zugleich spartanischen Bild zu erkennen (wenn nicht, neige den Kopf etwas nach links, um den Hasen, etwas nach rechts, um die Ente zu sehen). Bestimmte Elemente wie Hals oder Auge sind für beide Tiere identisch. Anderen Elementen hingegen kommt zwar eine Funktion zu, sie spielen aber jeweils unterschiedliche Rollen. So ist offensichtlich der Hasenmund eine Beule des Entenkopfes, dessen Schnabel wiederum die langen Löffelohren des Hasen bilden. Wittgenstein spricht hier von 〈Aspekten〉 desselben Bildes. ${ }^{3}$ Was ist gemeint?; zunächst, dass das Bild hermetisch-mehrdeutig ist, d.h. die Aspektambivalenz verdankt sich dem Bild selbst und nicht externen Zusatzinformationen (obgleich der Hintergrund relevant sein kann, s.u.); sodann, dass das Bild uneindeutig-stabil ist, d.h. der eine Aspekt löst den anderen nicht ab oder verdrängt ihn, sondern alle Aspekte - trotz möglicher Präferenzen - bestehen gemeinsam; endlich, dass das Bild funktional-vollständig ist, d.h. jedes Element muss in allen Aspekten eine Rolle spielen und keines darf in einem Aspekt (oder mehreren) vakant bleiben. Hermetische Mehrdeutigkeit, uneindeutige Stabilität und funktionale Vollständigkeit konstituieren nun unterschiedliche Aspekte eines Bildes als kognitive Möglichkeiten - was aber führt zu ihrer wirklichen Perzeption? Gehen wir von den Aspekten zum Aspekte-Sehen über. Ich möchte das anhand von vier Merkmalen tun:

(1) Aspektwechsel: Schauen wir auf den obigen Hasen-Enten-Kopf (H-E-Kopf), liegt die Frage nahe, welchem Zusammenhang sich der Wechsel der Aspekte verdankt. Jeder wird aus eigener Erfahrung wissen, dass dieser Aspektwechsel oft reziprok abläuft: Man erfasst den einen Aspekt, konzentriert sich dann darauf, den anderen auszumachen, und ist dies gelungen, mag einem der erste wieder entglitten sein, ohne ihn gänzlich zu verlieren. Was also geht hier vor sich? Klar ist, dass dieses shifting weder auf einen wie auch immer gearteten zusätzlichen empirischen Input, noch auf eine objektive Veränderung, eine gewandelte Zusammensetzung des Bildes zurückzuführen ist. Attraktiver wirkt das Angebot, den Aspektwechsel auf Deutungsverschiebungen zurückzuführen. Nun benötigt Deuten erstens Zeit (episodisch) und hängt zweitens stark vom Deutenden $\mathrm{ab}$ (relativ). Jedoch entsprechen Episodizität und Relativität kaum der gleichsam plötzlichen Emergenz der Aspekte sowie ihrer bereits erwähnten, die Abhängigkeit von externen Faktoren stark eingren-

L. Wittgenstein, Philosophische Untersuchungen, Teil II, xi, bes. S. 518-530 sowie Bemerkungen \& Letzte Schriften über die Philosophie der Psychologie, passim. 
zenden Hermetik. Daher drückt Wittgenstein seine Bedenken gegenüber diesem Deutungsvorschlag (im doppelten Sinn) wie folgt aus:

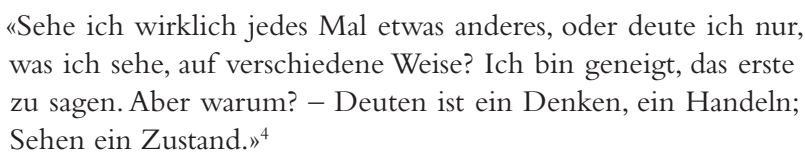

Was aber dann? Mit dem Gedanken der funktionalen Vollständigkeit ist, wie gesehen, der Umstand angesprochen, dass jedem Element innerhalb aller möglichen Aspekte eine bestimmte Rolle zuzukommen hat. Dass es sich so verhalten muss, ist ebenso notwendig, wie offen bleibt, welche konkrete Rolle ein Element dabei einnimmt. Solange diese Rolle sozusagen im Fluss und die Organisation der Elemente nicht festgestellt ist, können wir unterschiedliche Einstellungen zur Figur einnehmen, welche schliesslich nicht mittels unserer Deutungen (aktiv), sondern durch die Aspekte des Bildes selbst justiert werden (passiv). Wir werden dann in bestimmter Weise auf die Figur antworten. ${ }^{5}$

(2) Bedingungen des Aspekte-Sehens: Ganz so einfach liegen die Dinge jedoch zwischen aktiver Deutung vs. passiver Perzeption nicht. Es lassen sich Bedingungen auf Seiten des Rezipienten benennen, die ein Aspekte-Sehen überhaupt erst ermöglichen, diesem förderlich sind oder es als relevant erscheinen lassen. (i) So mag der H-E-Kopf mit dem performativen Akt des spielerischen AspekteSehens verwoben sein, dass man unhinterfragt auf die Suche nach den darin «versteckten` Tieren geht. Doch solche Verbindungen zwischen einem Bild und dem Umgang mit ihm sind keineswegs selbstverständlich. Ein (Gegen)Beispiel: In gewisser Hinsicht können zwei unterschiedliche Beschreibungsweisen eines Ölgemäldes Aspekte dieses Kunstwerkes genannt werden: Es könnte unter ästhetischen Gesichtspunkten thematisch werden, aber auch unter chemischen, welche der Zusammensetzung verwendeter Farben nachgingen. Wie also das Verhältnis zwischen Gegenstand und seiner Handhabung zu gestalten ist, hängt wesentlich vom Kontext ab, in dem sich der Rezipient befindet. (ii) Dass des Weiteren Erfahrung und Vorstellungskraft dem gelingenden Aspekte-Sehen zugute kommen, ist nur dann trivial, wenn man es auf H-E-Köpfe (und dergl.) beschränkt. Erweitert man den Gegenstandsbereich etwa auf moralische oder religiöse Fragen, verbindet sich die Erfahrung,

4 Ebd., 550

5 Auf dieses Moment macht bes. M. McGinn aufmerksam: Wittgenstein and the Philosophical Investigations, London 1997, $202 \mathrm{f}$. 
etwas auch anders sehen $\mathrm{zu}$ können, vielleicht $\mathrm{zu}$ müssen, und daran die Imagination zu schulen, bestenfalls mit Dialogfähigkeit und Toleranz. (iii) Überdies generiert das Aspekte-Sehen kein neues Wissen, sondern setzt voraus, dass - um einen Aspekt im Bild zu erkennen - dieser Aspekt unabhängig vom Bild, also aus anderen Zusammenhängen bekannt ist. Diese Bekanntschaft kann unterschiedliche Gestalt annehmen: visuelle Vertrautheit oder (damit gewöhnlicher-, nicht aber notwendigerweise verknüpft) begriffliche Kompetenz. ${ }^{6}$ Warum diese Differenzierung? Nun, man kann einen bestimmten Gegenstand, zum Beispiel ein filigranes Werkzeug (von dem man nicht wissen muss, dass es eines ist) repräsentieren, ohne über den entsprechenden Begriff zu verfügen (thetisch hinzugefügt sei: ohne überhaupt begrifflich denken zu müssen - ein weites Feld: Denke, lieber Leser, an unseren Hasen und die Ente, die keine 〈Hasen〉 und 〈Enten〉 besitzen). Umgekehrt ist es vertrackter, denn wir haben zwar häufig Begriffe, ohne die zugehörigen Gegenstände je gesehen $\mathrm{zu}$ haben - u.a. deshalb, weil nicht alle Begriffe auf Gegenstände referieren. Mit Bezug auf das Aspekte-Sehen fragt sich jedoch, wie ein Aspekt erkannt werden soll, den man nur konzeptuell kennt (in nomine), aber nie sinnlich erfahren konnte (in imagine). Versteht man 〈Aspekt〉 allein gegenständlich, stellt sich dieses Problem tatsächlich. Das muss man aber nicht tun; stattdessen werden wir den Begriff des Aspektes weiter fassen, so dass er nicht nur kognitive, sondern auch konzeptuelle Möglichkeiten abdeckt (s. [3] \& Abschnitt II).

(3) Aspektblindheit: Die drei Bedingungen des Kontextes (i), der Erfahrung (ii) und der Bekanntschaft (iii) bewegen sich offensichtlich auf ganz unterschiedlichen Ebenen: (i) benennt eine Relevanzbedingung, (ii) eine Bedingung, die dem Aspekte-Sehen dienlich ist, sozusagen eine Erleichterungsbedingung, allein (iii) macht auf eine Ermöglichungsbedingung und damit auf eine conditio sine qua non aufmerksam. Kurz: Das Sehen von Aspekten ist ohne (i) und (ii) möglich, nicht aber ohne (iii). Dies hat zur Folge, dass es umgekehrt unterschiedliche Grade von Aspektblindheit gibt; denn die naheliegende Idee, wonach die Nichterfüllung mindestens einer der drei Bedingungen zur Unfähigkeit führt, einen bestimmten Aspekt zu sehen, folgt nicht einem derartigen Automatismus. Dieser stellt sich allein bei der notwendigen Bedingung (iii) ein, sodass ihre Abwesenheit tatsächlich in die Aspektblindheit mündet.

Zunächst: Entweder man sieht den Aspekt oder man sieht ihn nicht - es gibt nicht so etwas wie eine〈Aspekt-Kurzsichtigkeit), der man

\footnotetext{
6 Vgl. K. von Stosch, Glaubensverantwortung in doppelter Kontingenz, Regensburg 2001,280 .
} 
mit entsprechenden Sehhilfen begegnet. Bleiben wir aber innerhalb der pathologisierenden Metapher der Blindheit, dann liegt die Frage nach möglichen Heilungen nicht fern. Dazu müssen wir jedoch unterschiedliche Krankheitsbilder auseinanderhalten. Wittgenstein ist allein am Aspekte-Sehen als kognitivem Akt interessiert - Blindheit wäre einer Sehstörung ähnlich. Nun versuchte ich dazu anzuregen, dieses Verständnis zweifach zu erweitern: So ging es unter (2) um konzeptuelle Möglichkeiten als Aspekte - Blindheit wäre semantische Armut; in der Einleitung deutete ich zudem (un)mögliche und notwendige Wege der Lebensführung und damit existentielle Aspekte an - Blindheit wäre lebensweltliche Verabsolutierung. Insbesondere auf die zweite und dritte der genannten Formen samt Heilung komme ich gleich zurück.

(4) Sehen und Sehen als...: Entgegen Wittgensteins Intention ist die Idee des Aspekte-Sehens von manchen Autoren generalisiert worden $\mathrm{zu}$ einer für jede sinnliche Wahrnehmung verbindlichen erkenntnistheoretischen These. ${ }^{7}$ Diese ist aber mit Vorsicht zu geniessen, was an folgender Unterscheidung deutlich werden mag. Schauen wir wieder auf den H-E-Kopf und leugnen, dass sich darin auch eine Ente sehen lässt, begingen wir tatsächlich einen Fehler. Dieses Bild nimmt in unserer Praxis einen Ort ein, der uns in gewisser Weise zwingt, die Aspekte aufzusuchen (obligates AspekteSehen). Zum Kontrast: An einer späten Stelle spricht Wittgenstein vom menschlichen Körper (und dessen Mienenspiel \& Gesten) als dem «beste[n] Bild der menschlichen Seele». ${ }^{8}$ Erlägen wir auch hier einem Fehler, wenn wir diesem interpretatorischen Akt (etwa in der Leugnung der Seele) nicht Folge leisteten? Offenbar nicht (fakultatives Aspekte-Sehen). Und schliesslich: Wir haben im gewöhnlichen Leben ununterbrochen Umgang mit Gegenständen, die derart in unsere Lebenspraxis eingebettet sind, dass sich in Bezug auf sie das «seeing-as» / «experiencing-as〉 gar nicht einstellt. Wie wir schon besprochen haben, kann man nur dann sinnvoll vorbringen, man sehe $x$ als $y$, wenn man gewahr ist, dass es neben $y$ weitere Kandidaten für das Sehen von $x$ gibt. In der Mensa, dem Restaurant oder auf der Grill-Party sehe ich hingegen meine Gabel nicht als Gabel an; ich verwende schlicht ein Essbesteck, das glücklicherweise seinen festen

\footnotetext{
7 Ganz bes. J. Hick, z.B. in: An Interpretation of Religion, New Haven 1989, 140 143, wobei Hick das seeing-as zum experiencing-as fortschreibt; kritisch dazu: M. Scott, Seeing Aspects, in: IJPR 44 (1998), 93-108.

8 Wittgenstein, PU II, S. 496; vgl. PU 283.357; sehr präzise und instruktiv: N. Fleming, Blick auf die Seele, in: E. von Savigny (Hg.), Ludwig Wittgenstein. Philosophische Untersuchungen, Berlin 1998, 239-268.
} 
Platz bei derartigen Zusammenkünften hat, von denen unglücklicherweise allzu häufig abstrahiert wird (sinnloses Aspekte-Sehen).

Natürlich liesse sich auch für das Sehen als... das Selbstanwendungsmanöver durchführen: Demnach stellte sich das Sehen als... als ein Sehen als... des Sehens heraus. Doch da dieser Zug keineswegs ‘obligat〉, eher 〈fakultativ〉 und für unsere Zwecke «sinnlos〉 ist, gehe ich sogleich - die bislang erarbeiteten Unterscheidungen anwendend - zum eigentlichen Anliegen über.

\section{Religiöser Glaube als Aspekte-Sehen?}

Dieser fragende Untertitel bezeichnet alles andere als einen religionsphilosophischen Dauerbrenner. Dazu hielt Michael Scott 1998 fest:

"What is surprising, however, is that with the exception of the work of John Hick, the notion [of seeing aspects] has received a comparatively rare and superficial treatment in philosophy of religion $[\ldots] . \iota^{9}$

Das hat sich in jüngster Zeit kaum geändert. Selbst bei den explizit an Wittgenstein anknüpfenden Theologen und Religionsphilosophen findet sich, soweit ich sehe, keine Stelle, in der dem Aspekte-Sehen systematisch nachgegangen worden wäre. ${ }^{10}$

Um dafür einen kleinen Beitrag zu leisten, gehe ich so vor, dass nochmals die vier erläuterten Momente des Aspekte-Sehens, nun mit Blick auf den religiösen Glauben, abgeschritten werden.

$\left(1^{\star}\right)$ Aspektwechsel: Bei der Betrachtung des H-E-Kopfes war deutlich, dass das shifting der Aspekte nicht auf eine objektiveVeränderung des Gesehenen zurückgeführt werden kann. Eher wird es unsere Einstellung zur Figur sein, die uns unterschiedliche Aspekte als deren interne Eigenschaften sehen lässt. Etwas technischer: Hase und Ente supervenieren auf den H-E-Kopf. Mögliche ontologische Fragen sind damit offensichtlich noch gar nicht berührt; denn wir könnten uns einen H-E-Kopf vorstellen, in dem 〈E〉 für 〈Einhorn〉 steht, sodass trivialerweise mit möglichen Aspekten (in imagine) nichts über deren wirkliches Vorkommen (in re) gesagt ist.

\footnotetext{
9 Scott, Seeing Aspects, s. Anm. 7, 93.

${ }^{10}$ Eine wenn auch eingegrenzte Verwendung findet das Aspekte-Sehen als exegetisches Vehikel bei M.J. Ferreira, die damit Kierkegaards Redeweise vom «Sprung» und (Paradox) reformuliert; siehe ihr Buch Faith and the Kierkegaardian Leap, Cambridge 1997.
} 
Diese Konstellation kommt jedoch nur dadurch zustande, dass unser Bild etwas bebildert, weshalb die Frage gestattet ist, ob das Abgebildete denn existiert. Parallelisieren wir nun das AspekteSehen mit religiösem Glauben, verhalten sich die Dinge anders. Die mehrdeutige Figur muss ersetzt werden durch die uns - ebenso mehrdeutig - umgebende Realität. Der Glaube (in einer bestimmten Ausprägung) nimmt die Position des auf diese Wirklichkeit supervenierenden Aspektes ein. Das dafür notwendige ontologische Inventar - 〈die Welt - ist bereits gegeben. Für diesen Zug drängen sich zwei Lesarten auf:

(a) Man könnte behaupten, dass, anders als beim H-E-Kopf, im Glauben tatsächlich etwas Neues entdeckt wird; das ontologische Inventar würde erweitert.

(b) Man könnte vorbringen, dass der Glaube uns am konstant gehaltenen ontologischen Inventar etwas sehen lässt, was wir extra fidem übergingen.

Im Rahmen des Aspekte-Sehens jedoch spricht einiges gegen die These des Realismus (a). Erinnern wir uns an die funktionale Vollständigkeit, nach der jedem Element in allen Aspekten eine Rolle zukommen muss, so ist dies gemäss (a) unmöglich: 〈Gott`spielt keine Rolle im dann funktional unvollständigen Aspekt des Unglaubens.

Wofür kann nun innerhalb von (b) diese Parallelisierung stehen? Wenn religiöser Glaube als Aspekt anzusehen wäre, dann das ZumGlauben-Kommen als Aspektwechsel. (b) verpflichtet uns überdies, darin nicht die Entdeckung eines wie auch immer gearteten matter of fact zu sehen (so ja [a]), sondern vielmehr die Gewinnung einer ganz neuen Einstellung zu dem, womit auch der Nicht-Glaubende, Ungläubige konfrontiert ist. Gerade wenn wir Wittgensteins Idee des Aspekte-Sehens auf religionsphilosophischem Terrain fruchtbar einbringen wollen, sollten wir seine Fokussierung auf kognitive Sachverhalte auf sich beruhen lassen und uns auf die vorgeschlagenen Erweiterungen, der konzeptuellen und der existentiellen Dimension des Aspekte-Sehens, konzentrieren. Was heisst das? Es heisst, dass die aspektwechselnde Gewinnung des Glaubens im einen Fall auf die (Rück)Gewinnung sprachlich-grammatischer Ressourcen hinausläuft (meaning), im anderen Fall auf die Bereitschaft bzw. das Geschenk, den Lebensvollzug unter einer bestimmten Perspektive, coram Deo, zu verstehen, vielleicht: verstehen zu müssen (significance). ${ }^{11}$ Ich komme darauf zurück.

${ }^{11}$ Daher gilt mit K. von Stosch: Nicht jeder Aspektwechsel ist die Installierung einer neuen Grammatik oder (ausschliessend gemeint) die Inkraftsetzung einer neuen 
$\left(2^{\star}\right)$ Bedingungen des Aspekte-Sehens: Drei Bedingungen trugen wir zusammen: die Relevanzbedingung (i: Kontext), die Erleichterungsbedingung (ii: Erfahrung) und die notwendige Bedingung der Bekanntschaft (iii: begriffliche Kompetenz \& visuelle Vertrautheit). $\mathrm{Zu}\left(i^{\star}\right)$ : Im ersten Abschnitt war (i) so aufgefasst, dass der Kontext entscheidet, ob (und inwiefern) ein Aspekt relevant ist; erinnert sei an das Ölgemälde: Meist ist der chemische Aspekt der Farbzusammensetzung für Museumsbesucher unwichtig. Diese Kontextualität hat hier nun ihre Grenzen; denn der Glaube ist unbedingt. ${ }^{12}$ Genauer: Der Glaube ist zwar in seinen Inhalten kontextabhängig, in seiner Relevanz jedoch nicht kontextgebunden. Gerade das Aspekte-Sehen kann verstehen helfen, dass und wie der (funktional-vollständige) Glaube uns alles anders zu sehen lehrt. Zu (ii $\star$ : Die (hier nicht im strengen Sinn zu verstehende) Erfahrungsbedingung lässt sich auch auf den religiösen Glauben anwenden - besser: Der Glaube ist die Erfahrung, etwas anders sehen zu können, und verdichtet sich zu der Gewissheit, es auf diese andere Weise, coram Deo, sehen zu müssen - nicht vergessend, dass es - darin dialogfähig und tolerant - mit ihm gemeinsam bestehende Aspekte gibt. Zu (iii ${ }^{\star}$ ): Der als diese bestimmte Erfahrung skizzierte Glaube geht mit einem Vokabular für das Verständnis unserer selbst, der Welt und Gottes einher, wobei visuelle Vertrautheit nicht abzielt auf eine 〈Bekanntschaft〉 mit einem exklusiven Sachverhalt oder einer «Superperson` (wiederum gegen [a]), sondern auf eine Form der Aufmerksamkeit, die den Glaubenden vom Nicht-Glaubenden unterscheiden wird (so mit [b]). Die begriffliche Kompetenz könnte dreifach ausgelegt werden als Bitte um das rechte Wort (Gebet), als Sich-Wiederfinden im Wort (Bekenntnis), als Suche nach dem treffenden Wort, einer «neue[n[ Sprechweise» ${ }^{13}$ (Theologie).

$\left(3^{\star}\right)$ Aspektblindheit: Mit dem Transfer des Aspekte-Sehens auf das religionsphilosophische Feld haben wir das traditionell zentrale kognitive Moment in den Hintergrund gerückt - das hat natürlich ebenso traditionelle Gründe: «homo est manifestus, deus autem invisibilis». ${ }^{14}$ Das Augenmerk ist daher auf konzeptuelle und existentielle Aspekte sowie nun die entsprechenden Blindheiten gerichtet. Letztere, so mag sich auch hier nahelegen, könnten wiederum mit

Perspektive; vielleicht könnte man genau das behaupten, verstünde man das «oder einschliessend (vgl. Glaubensverantwortung, s. Anm. 6, 281).

12 Das kann man, so denke ich, unabhängig von einer Innen- oder Aussenperspektive auf den Glauben behaupten.

${ }_{13}$ Wittgenstein, PU 400

${ }^{14}$ M. Luther, WA 3, 302. 
der Nichterfüllung der unter $\left(2^{\star}\right)$ erläuterten Bedingungen erklärt werden. Genauer besehen fällt jedoch $(i \star)$ als conditio heraus, zumal die Pointe gerade in der Asymmetrie zwischen relativer Relevanz von Aspekten (in [i]) und unbedingter Relevanz des Glaubens als Aspekt (in $\left[i^{\star}\right]$ ) bestand. Die unter $\left(i^{\star}{ }^{\star}\right)$ verhandelte, zu Dialog und Toleranz befreiende Erfahrung des Anders-Sehen-Könnens kann sich hingegen sehr wohl förderlich auf das religiöse Aspekte-Sehen auswirken, während uns deren Absenz etwas in unserem Leben nicht sehen lassen mag. Doch ohne ein mit Lebensformen verwobenes Vokabular, also (iii^), ist es unmöglich, den Glaubensaspekt erblicken zu können - denken wir an die eben erwähnte Trias des Wortes in Gebet, Bekenntnis und Theologie. Unglaube wäre folglich die Unmöglichkeit, diesem semantischen Aspekt als Moment existentiellen Selbstverständnisses einen Ort im Leben geben zu können. «Offenbarung〉 kann das Geschehen genannt werden, diese Unmöglichkeit aufzuheben.

Nur, sollten wir von Offenbarung als «Heilung〉 der Aspektblindheit sprechen und damit Formen des Unglaubens, des Nicht-GlaubenKönnens in die Nähe einer Krankheit rücken? Metaphern führen bekanntlich ihr Eigenleben und manchmal versagen sie. Im Gegensatz zum Unvermögen, den Hasen im H-E-Kopf zu erkennen, ist der Ungläubige in gewisser Weise 〈bekannt〉 (nicht unbedingt vertraut) mit der religious option (in Abwandlung von Ch. Taylors «secular option`). Nicht eine Sehstörung liegt hier vor, sondern eine existentielle Unmöglichkeit, einem Aspekt im Lebensvollzug Raum zu geben, so wie es für einen westeuropäischen Christen höchstwahrscheinlich unmöglich ist, einen afrikanischen Stammeskult als ernstzunehmenden Aspekt zu begreifen. Hineinwachsen und neu Sprechen-Lernen bilden daher die unter (iii ${ }^{\star}$ ) genannte Bedingung. Das schliesst die plötzliche Emergenz des Glaubensaspekts als Offenbarung mit ein nicht aber als Heilung einer Krankheit, sondern als Unterbrechung der Gesundheit.

$\left(4^{\star}\right)$ Sehen und Sehen als...: Lange Zeit war der christliche Glauben in unserer Region kein Aspekt; denn - denke, lieber Leser, an die dafür notwendige Pluralität - er war konkurrenz-, alternativlos. Heute hat sich offenbar die Selbstverständlichkeit des Glaubens verflüchtigt. Es existiert die 〈secular option〉 und erst mit ihr der Glaube als Aspekt, d.h. als sinnvolles Aspekte-Sehen. Schauen wir, ob der religiöse Glaube zudem ein obligates oder doch fakultatives Aspekte-Sehen darstellt. Als Beispiel für obligates Aspekte-Sehen diente der H-E-Kopf, weil man, soll ein kognitiver Fehler umgangen werden, beide Aspekte erkennen muss. Hingegen ist man offen- 
sichtlich nicht in gleicher Weise gezwungen, Glaube und Unglaube als dazu äquivalente Aspekte anzuerkennen. Interessanterweise gilt das aber nur für den Nicht-Glaubenden: Für ihn ist der Glaube kein Aspekt; anders der glaubende Mensch: Er muss fähig sein, beide Aspekte zu erkennen. ${ }^{15}$ Als Beispiel für fakultatives Aspekte-Sehen fungierte eine mögliche Interpretation (Wittgensteins Blick auf die Seele)), die man teilen kann, aber nicht muss. Ist also der Glaube eine mögliche, nicht notwendige Interpretation «der Welt»? Auch hier kommt es darauf an, von welchem Punkt man schaut: Aus der Innerperspektive des Glaubens muss man die Frage wohl verneinen; denn dass Jesus der auferstandene Christus ist, bildet eine Gewissheit, nicht eine Option (Notwendigkeit). Aus der externen Perspektive gibt es diese Gewissheit nicht einmal als Option (Unmöglichkeit). Wohl aber gibt es die disparaten Phänomene des Zweifels vor jeder 〈Entscheidung〉 (Möglichkeit).

Wenn der religiöse Glaube in diesem Sinn als ein sinnvolles, obligates, aus der internen Sicht nicht-fakultatives Aspekte-Sehen anzusprechen ist, müsste er zugleich als Sehen als... verstanden werden: Im Gegensatz zur selbstverständlichen Benutzung der Gabel bei Grill-Partys ist der religiöse Glaube aussergewöhnlich, da der Glaubende stets zum uneindeutig-stabilen Aspektwechsel zwischen Glaube und Unglaube fähig sein muss.

\section{Folgerungen - Grenzen}

Ich hatte mich bemüht, religiösen Glauben durch das (auch hier) exegetischeVehikel des Aspekte-Sehens zu charakterisieren. Bewusst hatte ich es in der Schwebe gehalten, dass der Glaube sowohl als ein eigenständiger Aspekt (neben anderen), wie auch als das AspekteSehen selbst (das Sehen als...) angesprochen werden kann. Beides aber gehört offenbar untrennbar zusammen: Ein nicht gesehener Aspekt ist sinnlos; ein nichts sehendes Aspekte-Sehen inhaltsleer. Religiöser Glaube ist in dieser Doppelung die existentielle Möglichkeit, mich selbst, den Nächsten und die uns umgebende Welt in einem bestimmten, in Lebensformen eingebetteten Vokabular zu verstehen - und aussergewöhnlich darin, neue Aspekte am Gewöhnlichen

\footnotetext{
${ }^{15}$ Dieses Gefälle kann man sich am Schöpfungsglauben verdeutlichen: Der Glaubende kann die Welt z.B. als creatio und als evolutiven Zusammenhang erfassen; dem Nicht-Glaubenden wäre nur letzteres möglich; vgl. P. Winch, Darwin, Genesis und Widersprüchlichkeit, in: ders., Versuchen zu verstehen, Frankfurt a.M. 1992, 183-192.
} 
zu erkennen, nicht Neues jenseits des Gewöhnlichen; denn: Einen Aspekt gibt es nur, wo es mindestens einen anderen Aspekt gibt.

Und eine Position wie diese gibt es wohl nur, wenn es gegnerische Positionen gibt. Von jenen sind dann zwei Einwände zu erwarten: der Relativismusverdacht sowie der Reduktionismusvorwurf. Auf der Linie des ersten Bedenkens liesse sich vorbringen, das Problem der Wahrheit (oder Inadäquatheit) eines Aspektes werde übergangen. Darauf möchte ich nur antworten, dass es um die Frage geht, ob sich ein Mensch in einer religiösen Tradition als konzeptuellem und darin existentiellem Aspekt angemessen wiederfindet - im Sich-Orientieren, im Sich-Verstehen. Auf der Linie der zweiten Kritik liesse sich fragen, ob der religiöse Glaube nicht sehr viel stärkere ontologische Verpflichtungen impliziere, als es von mir nahe gelegt wurde. Darauf möchte ich nur antworten, dass sich der hiesige Vorschlag - in Betonung der besprochenen hermetischen Mehrdeutigkeit - gerade als Reaktion auf das Scheitern derartiger ontological commitments versteht.

Die Grenze der Parallelisierung des religiösen Glaubens mit dem Sehen von Aspekten mag an einem anderen Ort liegen. Wenn beide Aspekte im H-E-Kopf erkannt sind, kann man den Aspekt-Hasen sporadisch aus dem Blick verlieren, ohne deshalb leugnen zu müssen, dass dieser ein sinnvoller Aspekt der Figur bleibt.Verliert jedoch ein Mensch seinen religiösen Glauben, gerät dieser Aspekt des Lebens weder einfach sporadisch aus dem Horizont, noch wäre weiterhin zuzugeben, mit einem sinnvollen Lebensaspekt konfrontiert zu sein. Doch genau dort, wo diese Grenze verläuft, können sich neue Aspekte auftun. Behalten wir kombinierenden Theologen dies nicht im H-Kopf, wird uns tatsächlich noch Hören und Sehen (als...) vergehen.

- Hartmut von Sass ist Assistent am Lehrstuhl für Systematische Theologie, Symbolik und Religionsphilosophie der Universität Zürich sowie wissenschaftlicher Mitarbeiter am Collegium Helveticum. 